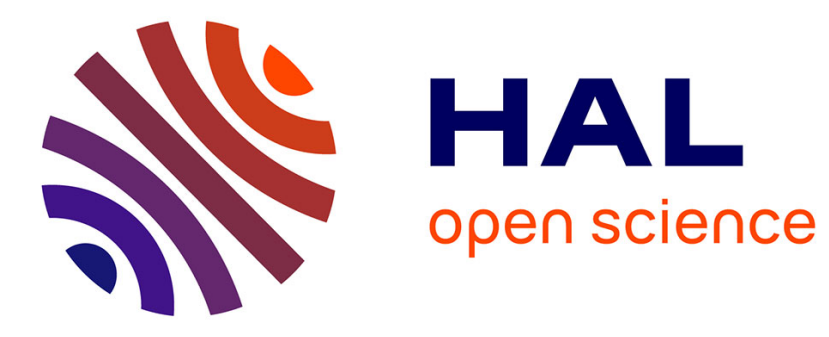

\title{
Concentration and Velocity Profiles in a Polymeric Lithium-ion Battery Electrolyte
}

Hans-Georg Steinrück, Christopher J Takacs, Hong-Keun Kim, David M

Mackanic, Benjamin Holladay, Chuntian Cao, Suresh Narayanan, Eric M

Dufresne, Yuriy Chushkin, Beatrice Ruta, et al.

\section{To cite this version:}

Hans-Georg Steinrück, Christopher J Takacs, Hong-Keun Kim, David M Mackanic, Benjamin Holladay, et al.. Concentration and Velocity Profiles in a Polymeric Lithium-ion Battery Electrolyte. Energy \& Environmental Science, 2020, 13 (11), pp.4312 - 4321. 10.1039/d0ee02193h . hal-03455773

\section{HAL Id: hal-03455773 https://hal.science/hal-03455773}

Submitted on 29 Nov 2021

HAL is a multi-disciplinary open access archive for the deposit and dissemination of scientific research documents, whether they are published or not. The documents may come from teaching and research institutions in France or abroad, or from public or private research centers.
L'archive ouverte pluridisciplinaire HAL, est destinée au dépôt et à la diffusion de documents scientifiques de niveau recherche, publiés ou non, émanant des établissements d'enseignement et de recherche français ou étrangers, des laboratoires publics ou privés. 


\title{
Concentration and velocity profiles in a polymeric lithium-ion battery electrolyte $\uparrow$
}

\author{
Hans-Georg Steinrück, (D) $\star^{\text {abc }}$ Christopher J. Takacs, (D) $\star^{\text {ab }}$ Hong-Keun Kim, (D) de \\ David G. Mackanic, (D) Benjamin Holladay, ${ }^{g}$ Chuntian Cao, (D) ${ }^{a}$ \\ Suresh Narayanan, (D) ${ }^{\mathrm{h}}$ Eric M. Dufresne, ${ }^{\mathrm{h}}$ Yuriy Chushkin, (D) ${ }^{\mathrm{i}}$ Beatrice Ruta, (D) ${ }^{\mathrm{ij}}$ \\ Federico Zontone,' Johannes Will, (DD k Oleg Borodin, (DD' Sunil K. Sinha, ${ }^{9}$ \\ Venkat Srinivasan ${ }^{\text {de }}$ and Michael F. Toney (iD *abm
}

\begin{abstract}
Predictive knowledge of ion transport in electrolytes which bridges microscopic and macroscopic length scales is imperative to design new ion conductors and to simulate device performance. Here, we employed a novel approach combining operando X-ray photon correlation spectroscopy, X-ray absorption microscopy, continuum modelling, and molecular dynamics simulations to probe the ion transport in a baseline polymeric lithium-ion battery electrolyte. In a Li/PEO-LiTFSI/Li symmetric cell under polarization, we determined and rationalized microscopic properties including local electrolyte velocities and ion correlations and connected this insight to measured and simulated macroscopic ion concentration gradients. By relating our results across length scales, we suggest a fairly concentration-independent transference number of about 0.2. Our study shows the broad applicability of operando X-ray photon correlation spectroscopy to the understanding of dynamic phenomena.
\end{abstract}

\begin{abstract}
Broader context
Quantitative understanding of ion and mass transport in liquid and polymeric electrolytes is at the heart of electrochemistry and many other energy related fields. Here, the goal is to accurately predict the concentration-dependent transport properties of the electrolyte and the resulting performance of an electrochemical device. In this context, one challenge is to unravel the transport phenomena occurring in concentrated solutions and determine the corresponding transport coefficients, as these are foundational to the performance of electrolytes in ion batteries. In this study, we employed a novel approach to understand the ion transport mechanism and evaluate transport coefficients, including transference numbers, in a baseline polymeric electrolyte. Specifically, we utilized in situ synchrotron X-ray photon correlation spectroscopy and X-ray microscopy to directly measure the electrolyte velocity and ion concentration profiles in a Li-polymer electrolyte cell. These were directly compared to calculations based on macroscopic concentrated solution theory, which contain the transference numbers as an input. Our results provide length- and time-scale bridging understanding of ion transport and we conclude a fairly concentration-independent transference number of 0.2 for a lithium bistrifluoromethanesulfonimidate/poly(ethylene) oxide electrolyte, which was rationalized via microscopic insight into the transport mechanism from molecular dynamics simulations.
\end{abstract}

\footnotetext{
${ }^{a}$ SSRL Materials Science Division, SLAC National Accelerator Laboratory, Menlo Park, California 94025, USA. E-mail: ctakacs@slac.stanford.edu

${ }^{b}$ SLAC National Accelerator Laboratory, Joint Center for Energy Storage Research (JCESR), Lemont, Illinois 60439, USA

${ }^{c}$ Department Chemie, Universität Paderborn, 33098 Paderborn, Germany. E-mail: hans.georg.steinrueck@uni-paderborn.de

${ }^{d}$ Argonne National Laboratory, Lemont, Illinois 60439, USA

${ }^{e}$ Argonne National Laboratory, Joint Center for Energy Storage Research (JCESR), Menlo Park, California 94025, USA

${ }^{f}$ Department of Chemical Engineering, Stanford University, Stanford, California 94305, USA

${ }^{g}$ Department of Physics, University of California, San Diego, La Jolla, California 92093-0319, USA

${ }^{h}$ X-Ray Science Division, Argonne National Laboratory, Lemont, Illinois 60439, USA

${ }^{i}$ ESRF - The European Synchrotron, 71 Avenue des Martyrs, 38043 Grenoble, France

${ }^{j}$ Univ Lyon, Université Claude Bernard Lyon 1, CNRS, Institut Lumière Matière, F-69622 Villeurbanne, France

${ }^{k}$ Center for Nanoanalysis and Electron Microscopy (CENEM), Friedrich-Alexander-Universitaät Erlangen-Nürnberg (FAU), IZNF, Cauerstr. 3, 91058 Erlangen, Germany

${ }^{l}$ Energy Storage Branch, Sensor and Electron Devices Directorate, U.S. Army Research Laboratory, Adelphi, MD 20783, USA

${ }^{m}$ Department of Chemical and Biological Engineering, University of Colorado, Boulder, CO 80309, USA. E-mail: michael.toney@colorado.edu

$\dagger$ Electronic supplementary information (ESI) available. See DOI: 10.1039/d0ee02193h

$\ddagger$ Equal contribution.
} 


\section{Introduction}

Understanding ion transport is of fundamental importance in natural sciences and engineering. Examples include biological ion pumps for life processes, ${ }^{1,2}$ porous membranes for separations, ${ }^{3,4}$ and electrodes and electrolytes in diverse electrochemical systems. ${ }^{5,6}$ While continuum level models can be used to predict macroscopic transport related behavior, the link to the microscopic transport mechanism is still to be realized. ${ }^{7}$ Connecting the corresponding spatial and temporal scales is hence a major goal towards quantitatively predicting how ions navigate through natural and designed systems. Electrochemical energy storage is one field where ions transport from one electrode to another through an electrolyte and is societally important towards the goal of carbon neutrality. Hence, knowledge of transport phenomena is necessary to both design new electrolyte and electrode materials and to accurately simulate the performance and safety of an electrochemical cell. This requires knowledge of the temperatureand ion concentration-dependent transport properties of an electrolyte, including the thermodynamic mean salt molal activity coefficient, the conductivity $(\sigma)$, the salt diffusion coefficient $(D)$, and the cation transference number $\left(t^{+}\right) .8,9$ The transference number is defined as the ratio of current carried by the cation to the total electric current, and is related to the mobilities of the anion and cation in dilute solutions and the Stefan-Maxwell diffusion coefficients in concentrated solutions. ${ }^{10}$ Importantly, these macroscopic quantities are intimately related to atomistic diffusion mechanisms.,11-14 For accurate cell simulations, the transport coefficients $\sigma, D$, and $t^{+}$must be known with high accuracy from experimental measurements, which is often complicated and necessitates many assumptions. Here, we present a novel approach in which we combine local operando measurements of electrolyte velocities and global measurements of ion concentrations with molecular scale simulations and continuum level modelling to relate molecular transport mechanisms to macroscopically observed transport phenomena. We anticipate that our method can be extended towards a variety of ion conducting materials.

The system we investigated is a polymer electrolyte for lithiumion batteries (LIBs). Polymer electrolytes are a safe alternative to commonly used flammable liquid organic electrolytes. ${ }^{5,15,16}$ However, their wide-spread adoption is hampered by typically poor ion transport properties. While the relevance of the transference number is sometimes overlooked, ${ }^{17}$ its importance in polymer electrolytes for LIBs with regards to the energy and power density was recognized in the early 1990s by Doyle, Fuller and Newman. ${ }^{18}$ The consequence of a transference number deviating from unity is concentration polarization, which has a strong impact on the LIB performance. A simple example is a Li/electrolyte/Li cell (anion blocking) under constant voltage conditions. Here, concentration polarization corresponds to a continued piling-up of anions and cations at the stripping electrode, and concurrent depletion near the plating side. A steady state is reached when the net motion of anions reaches zero, which is the condition that the diffusion force acting on anions due to the concentration gradient is equal to the migration force from the electric field (in the absence of convection). At high concentrations, volume conservation has the additional consequence of solvent mass transport upon salt mass transport. ${ }^{19}$ A conceptualization of mass transport phenomena occurring during polarization of a $\mathrm{Li}$ / electrolyte/Li symmetric cell is shown in Fig. 1(a). The concentration polarization is easily observed as the decrease in current upon constant voltage cell polarization until a steady-state current is reached. ${ }^{20}$ This increase in electrolyte resistance for ion conductors with non-unity transference number ultimately limits batteries' achievable rates, critical currents, and cutoff voltages (due to overpotentials), reducing the usable energy and power density. Hence, the transference number is an essential descriptor of ion transport. Nevertheless, researchers still argue about transference number values, even in baseline systems such as lithium bistrifluoromethanesulfonimidate (LiTFSI) in poly(ethylene oxide) (PEO). ${ }^{21}$ The transference number can be measured via the steady-state Bruce-Vincent polarization method, ${ }^{22,23}$ and the Balsara-Newman method, ${ }^{24}$ as well as pulsed field gradient NMR (pfg-NMR) ${ }^{25}$ and pulsed field gradient electrophoretic NMR (e-NMR). ${ }^{21}$ Despite extensive efforts towards unified results of these approaches, in particular as a function of ion concentration, a clear picture has not yet emerged.

Towards this end, we developed an alternative approach towards determining the ion transport properties. Specifically, we directly and operando measured precise microscopic and macroscopic physical properties of the electrolyte upon cell polarization in a Li/electrolyte/Li cell, combined this with calculations via concentrated solution theory continuum modelling (CM), and rationalized our findings with microscopic insight from molecular dynamics (MD) simulations. We utilized a well-studied benchmark model system electrolyte consisting of PEO and LiTFSI at $\mathrm{a} \mathrm{Li}^{+}$to EO molar ratio of $r=0.1$. Under constant voltage polarization, we directly measured the velocity associated with the electrolyte and ions via heterodyne synchrotron X-ray photon correlation spectroscopy (XPCS), and the TFSI $^{-}$concentration gradient from electrode to electrode via X-ray absorption microscopy (XAM). This novel approach is conceptualized in Fig. 1(b). The significance of our results lies in the unification of microscopic and macroscopic predictions from simulation with experimental measurements as well as the self-consistent determination of a concentrationindependent transference number of approximately 0.2 . Our study paves the way for further length- and time-scale bridging understanding of ion transport.

\section{Experimental: approach - experimental and modeling velocity and concentration profiles}

The electrolyte velocity measurements were conducted using $\mathrm{XPCS}^{26,27}$ in the heterodyne modality, ${ }^{28-31}$ as illustrated in Fig. 1(b). XPCS measures the time evolution of the coherent scattering pattern (speckle pattern), which encodes the sample dynamics. In heterodyne XPCS, a static reference scattering signal is mixed with the dynamic scattering signal of the polymer electrolyte (details in the ESI $\dagger$ ). The resultant phase shift in the coherent scattering due to the constant velocity motion of the sample with respect to a fixed reference yields 
(a)

XAM: local concentration

$\mathrm{CM}$ : local concentration, instantaneous velocity

MD: molecular scale transport

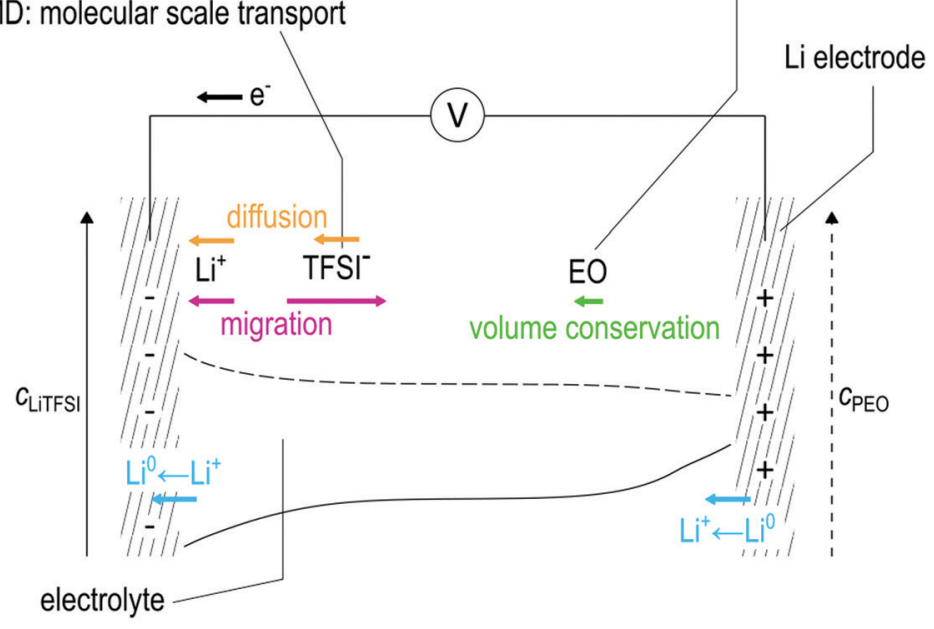

(b)

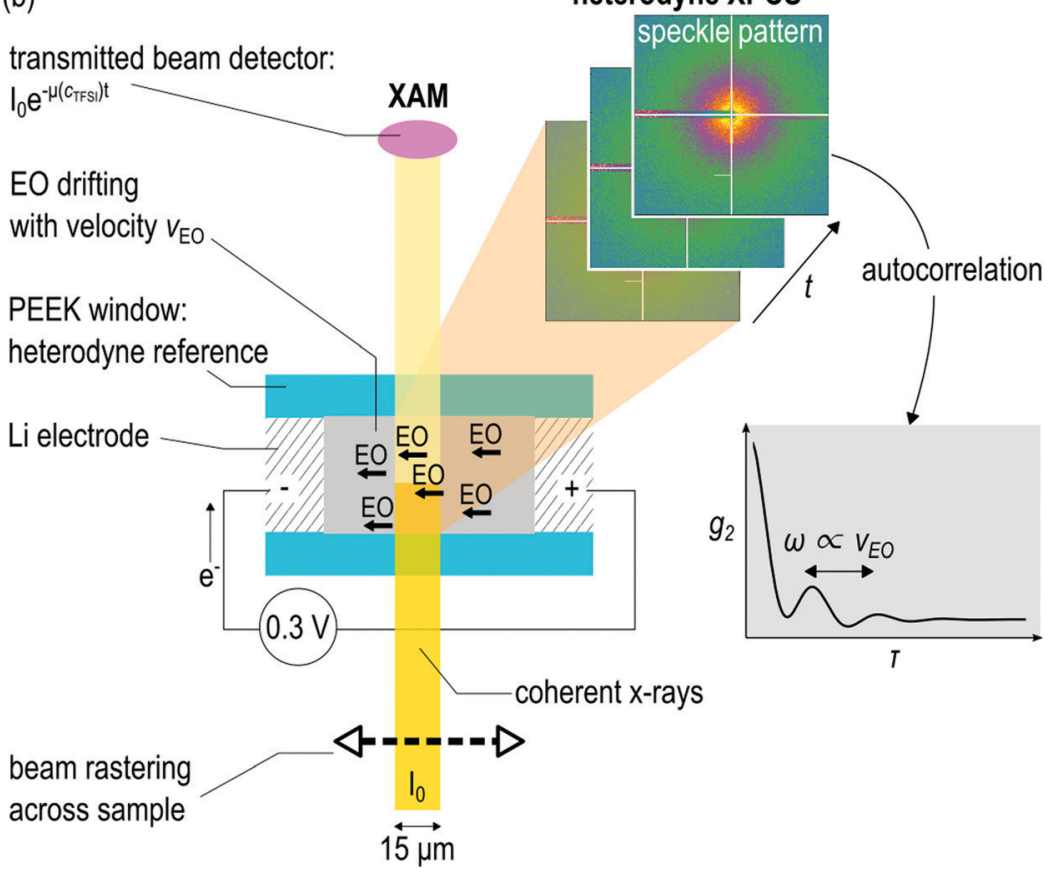

Fig. 1 Conceptualization of the problem and our approach. (a) Illustration of mass transport in an electrochemical symmetric cell, in which Li electrodes are separated by an electrolyte made up from ethylene oxide monomers (representing the PEO polymer), $\mathrm{Li}^{+}$cations, and TFSI ${ }^{-}$anions. Upon application of an electric field across the cell, $\mathrm{Li}^{+}$ions migrate in the electric field to the negative electrode, whereas $\mathrm{TFSI}^{-}$ions migrate to the positive electrode (magenta arrows, the length of which indicates relative velocities). As $\mathrm{Li}^{+}$is produced at the positive electrode and consumed at the negative electrode

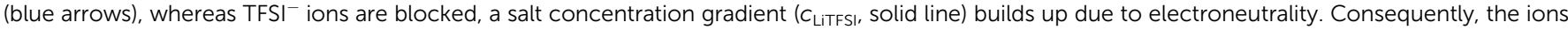
feel a diffusion force towards the negative electrode from the concentration gradient (yellow arrows). When the migration and diffusion forces acting upon TFSI ${ }^{-}$ions are equal, a steady state is reached, and there exists no TFSI ${ }^{-}$mass transport. At high salt concentrations, EO monomers are forced to the negative electrode due to mass conservation (green arrows), resulting in a concentration gradient in PEO ( $C_{\text {PEO, }}$ dashed line). The EO velocity scales with the molar volumes of the salt and EO. The macroscopic concentration gradient is measured by XAM and predicted by our continuum model, whereas our MD simulations unravel the molecular scale transport mechanism. TFSI ${ }^{-}$velocities can be directly correlated to the measured EO velocities by XPCS and predicted by our continuum model. (b) Schematic of the cell design (technical drawing in Fig. S1, ESI $\dagger$ ) and experimental setup to measure the EO velocity and TFSI ${ }^{-}$concentration profiles at $90{ }^{\circ} \mathrm{C}$; not to scale. The heating setup is neglected for simplicity. The lithium electrodes are separated by $3 \mathrm{~mm}$, and are connected to a potentiostat with a potential of $0.3 \mathrm{~V}$ applied between the electrodes. Heterodyne XPCS: the speckle pattern resulting from scattering of a coherent X-ray beam is recorded as a function of time; the beamline producing the coherent X-ray beam is described in ref. 53 . In the scenario of EO moving at a constant drift velocity, the heterodyne autocorrelation function shows oscillations, the inverse period of which is proportional to the EO velocity. XAM: The transmitted beam intensity is recorded as a function of time. Spatial resolution across the channel is achieved by rastering the $15 \mu \mathrm{m}$ beam across the channel. 
oscillations in the auto-correlation function $g_{2}(\mathbf{q}, \tau)$, which correlates the intensity in a given pixel at time $t$ to that at $t+\tau$; here, $\mathbf{q}$ is the scattering vector and $\tau$ is the delay time. The heterodyne auto-correlation function with heterodyne fraction $h$ and sample velocity vector $\mathbf{v}$ is given by

$$
\begin{aligned}
g_{2}(\mathbf{q}, \tau)=1 & +\beta(1-h)^{2}+h^{2} \beta \mathrm{e}^{-2\left(\frac{\tau}{\tau_{0}(q)}\right)^{\gamma}} \\
& +2 h(1-h) \beta \cos (\omega \tau) \mathrm{e}^{-\left(\frac{\tau}{\tau_{0}(q)}\right)^{\gamma}}
\end{aligned}
$$

with

$$
\omega=\mathbf{q} \cdot \mathbf{v}=q v \cos \chi
$$

Here $\chi$ is the angle between the scattering and velocity vector, ${ }^{28,32} \beta$ is the coherence factor, and $\tau_{0}$ is the system relaxation time. The relaxation is modelled by a stretched exponential decay with stretching factor $\gamma$. Eqn (2) allows for the rescaling and collapse of all correlation functions as functions of $\mathbf{q}$ and $\tau$ into a single master curve as shown in Fig. 2. Fig. 2(a) shows $18 \chi$-dependent correlation functions at a single $q$ and the corresponding fits to eqn (1); these data were obtained at beamline 8-ID-I at the Advanced Photon Source. The $\cos \chi$-dependence of the derived oscillation frequencies $\omega$ is shown in Fig. 2(c) together with a fit to eqn (2). The agreement between the data and the model allows us to calculate $\chi$-scaled correlation functions at each $q$, which are shown in Fig. 2(b) together with fits to eqn (1). The $q$-dependence of the derived $\omega$ is shown in Fig. 2(d). The observed linearity allows us to apply an additional
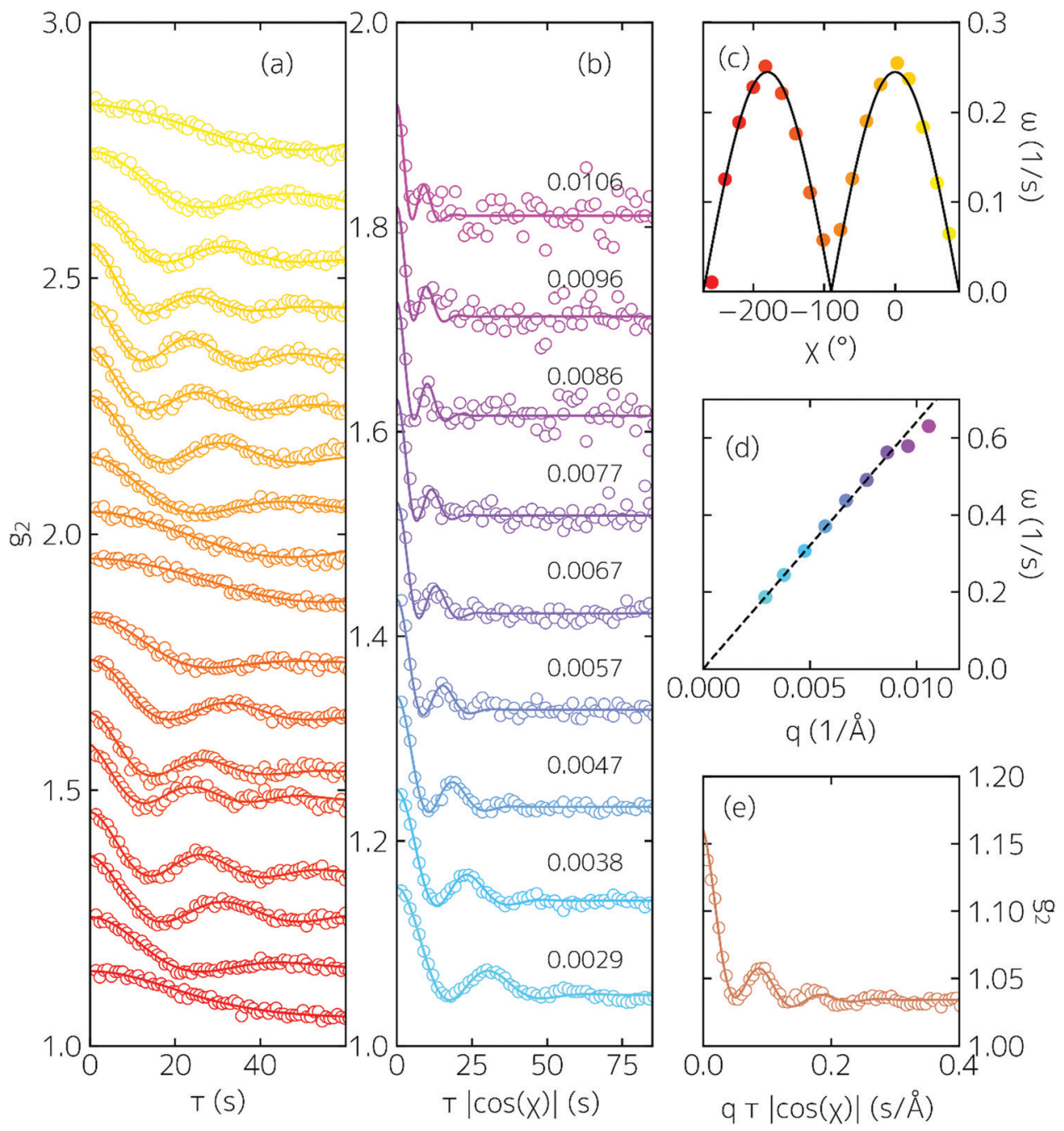

Fig. 2 XPCS analysis. (a) Measured auto-correlation functions (markers) as a function of delay time $\tau$ for different $\chi$-values at a $q$-value of $0.0038 \AA$ (corresponding to scattering vectors $q$ within the second innermost ring in Fig. S2, ESI $\dagger$ ), and model fits (lines) to eqn (1). The curves are vertically shifted for clarity. (c) Frequency parameters $\omega$ derived from the fits in (b) (markers) as a function of $\chi$ and the fit (line) to eqn (2) (colors correspond to the colors in (a)). (b) $\chi$ scaled correlation functions (markers) for the first nine $q$-values (indicated on top of each curve in $\AA^{-1}$ ) and model fits (lines) to eqn (1). The curves are vertically shifted for clarity. (d) Frequency parameters $\omega$ derived from the fits in (b) (markers) as a function of $q$. The line is a guide to the eye showing the expected linear behavior and a zero intercept (colors correspond to the colors in (b)). (e) $\chi$ - and $q$-scaled master correlation function (markers) collapsed from 72 individual correlation functions and the model fit (line) to eqn (1). 
linear $q$-scaling, yielding a single global master $q$ - and $\chi$-scaled correlation function (Fig. 2(e)), which shows oscillations, the inverse period of which corresponds to the velocity (details in the ESI $\dagger$ ).

Electrolyte velocities were measured operando during approximately 1000 minutes of polarization of a Li/PEO-LiTFSI/Li symmetric cell at a constant voltage of $0.3 \mathrm{~V}$ at $90{ }^{\circ} \mathrm{C}$ using a specially designed cell with the electrodes separated by $3 \mathrm{~mm}$ (details in the ESI $\dagger$ ). Position sensitivity was achieved by utilizing a $15 \mu \mathrm{m}$ X-ray beam positioned at different locations across the channel. In addition, during the same polarization experiment, XAM was utilized to measure the TFSI $^{-}$ion concentration gradient across the channel. For this purpose, the sample was raster-scanned across the channel, and the transmitted X-ray intensity was recorded, which was related to the concentration via LambertBeer's law, making use of the significantly higher absorption cross section for $\mathrm{TFSI}^{-}$compared to PEO (details in the ESI $\dagger$ ). The velocity and concentration gradients were then compared to our continuum modelling, where we employed concentrated solution theory, a mathematical one-dimensional model by Newman ${ }^{9}$ with a macro-homogenous assumption of the electrolyte (details in the $\mathrm{ESI} \dagger$ ). These macroscopic insights into the transport phenomena were coupled to the microscopic insights from MD simulations employing the Wohde-Roling-formalism. ${ }^{33}$

To compare our measured concentration gradients and electrolyte velocities to those predicted by CM, we chose two transport coefficients from recent literature. The first set (denoted RS transport coefficients) corresponds to values obtained by Rosenwinkel/Schönhoff $^{21}$ via the model-free approach of measuring the electrophoretic mobilities of the ions via e-NMR. The second (denoted PNB transport coefficients) corresponds to values obtained by Pesko et al. ${ }^{25,34}$ via the Balsara-Newman method. We note in passing that the former method yields similar values to those obtained via the Bruce-Vincent method and pfg-NMR. ${ }^{21,25,35,36}$ The rationale behind our choice lies in the large differences in the transference number of RS transport coefficients and PNB transport coefficients close to $r=0.1$, making our approach particularly sensitive to providing insight into the controversy regarding transference numbers around $r=0.1$. We point out that the effective transference numbers used in the calculations vary with time and position due to their concentration dependence (the concentration changes by $\sim 50 \%$ upon several hours of polarization); accordingly, we effectively probe a large range of concentrations from about $r=0.04$ to $r=0.16$, even though we studied only a single starting concentration. This variation is insignificant in the case of RS transport coefficients, whereas it is significant in the case of PNB transport coefficients. The exact transport coefficients used in our calculations are tabulated in Table S3 (ESI $\dagger$ ). As the referenced studies were performed in the conductive temperature-regime of PEO-LiTFSI at $90{ }^{\circ} \mathrm{C}$, above its glass transition and melting temperature, ${ }^{15}$ we also chose $90{ }^{\circ} \mathrm{C}$.

\section{Results and discussion}

Fig. 3(a) shows the experimentally measured current density (black line) upon $0.3 \mathrm{~V}$ polarization and the corresponding prediction from CM using input RS transport coefficients (blue line) and PNB

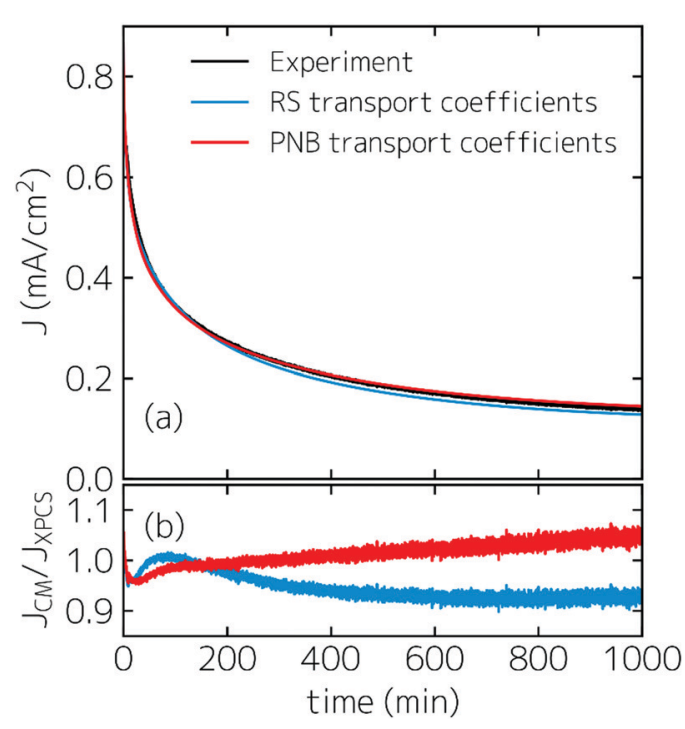

Fig. 3 Electrochemistry. (a) Experimental current density measured upon constant voltage $(0.3 \mathrm{~V})$ polarization of the Li/LiTFSI-PEO/Li symmetric cell of a channel length of $3 \mathrm{~mm}$ (black line). Continuum model predicted current density under conditions identical to the experiment using RS transport coefficients ${ }^{21}$ (blue) and PNB transport coefficients ${ }^{25,34}$ (red). (b) Continuum model predicted current density (using RS transport coefficients $^{21}$ (blue) and PNB transport coefficients ${ }^{25,34}$ (red)) divided by the experimental current density.

transport coefficients (red line). The overall shape of the curve is well reproduced by the model calculations. A more detailed comparison is illustrated in Fig. 3(b) where the ratios between the predicted and measured current densities are plotted. The measurements and prediction are within $10 \%$ over the entire polarization time independent of the model. While there are subtle shape changes during the first approximately 200 minutes, both predictions have a fairly constant difference from the measurement. We note that for an exact comparison, the interfacial resistance (e.g. due to nm-scale interfacial film formation upon TFSI $^{-}$reduction ${ }^{37-40}$ ) would need to be subtracted; it can be expected that bulk contributions are, however, dominant given the $3 \mathrm{~mm}$ channel thickness. ${ }^{25}$ Nevertheless, it is apparent that a comparison of the current densities alone does not allow for distinguishing between the two chosen transport coefficient models, even though the transference numbers are vastly different (see Table S3, ESI $\dagger$ ). To compare these two parameter-sets more rigorously and sensitively, we now examine the measured concentration polarization (via XAM) and ion velocities (via XPCS) compared to those predicted by CM. We note that XAM was inspired by optical methods of determining ion concentrations ${ }^{41-45}$ and quantitative determination of ion concentration gradients using X-ray phase contrast imaging. ${ }^{46}$

The XAM-derived concentration profiles (markers) upon cell polarization at $0.3 \mathrm{~V}$ are shown in Fig. 4(a) and (b) together with the profiles derived from CM (solid lines) (see Fig. S9, ESI, $\dagger$ for a false-color plot of the time-position dependence); these XAM data were obtained at beamline 8-ID-I at the Advanced Photon Source. Fig. 4(a) corresponds to simulation results using RS transport coefficients, whereas (b) corresponds to 


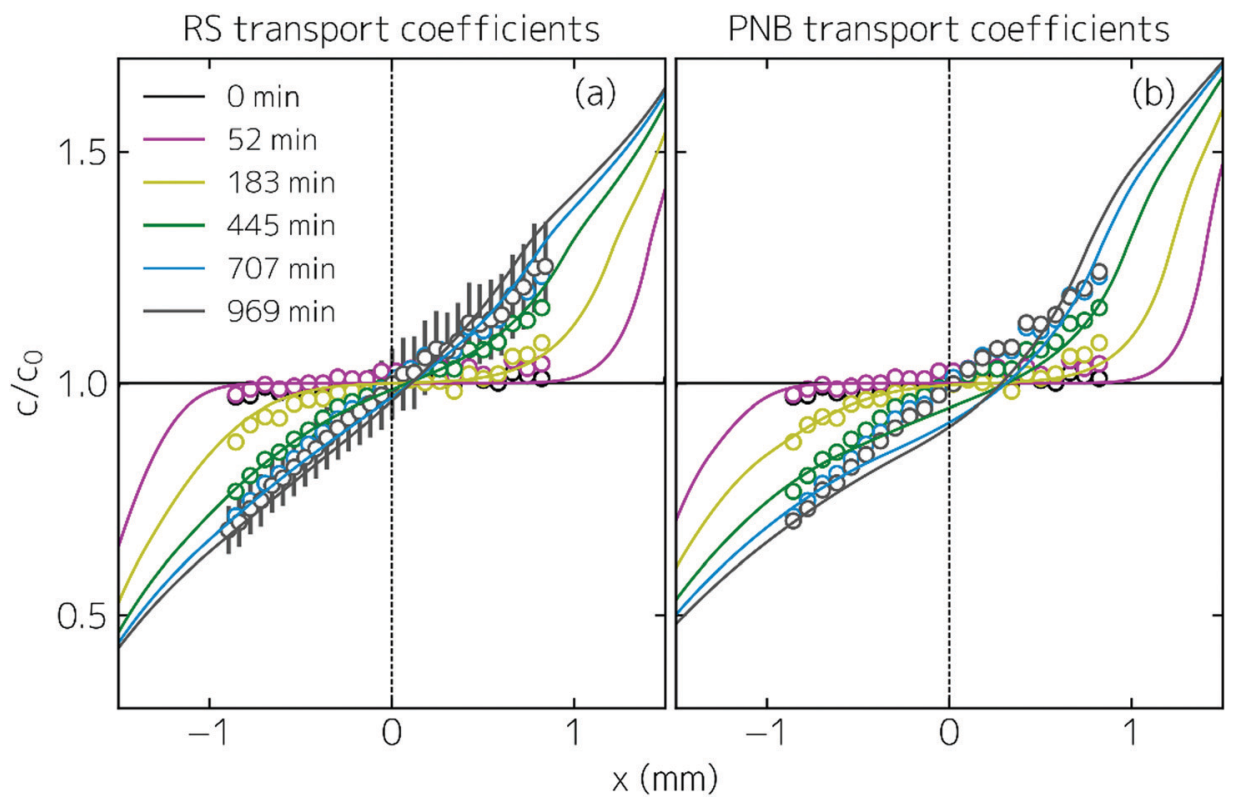

Fig. 4 Concentration polarization. (a) Experimental $\mathrm{TFSI}^{-}$concentration (markers) for different times measured upon constant voltage (0.3 V) polarization of the Li/LiTFSI-PEO/Li symmetric cell of a channel length of $3 \mathrm{~mm}$, and the TFSI ${ }^{-}$concentration predicted from the continuum model under conditions identical to the experiment using RS transport coefficients ${ }^{21}$ (lines); experimental error-bars are only shown for the curve at 969 min. (b) Same as (a) using PNB transport coefficients. ${ }^{25,34}$

PNB transport coefficients. The experimental profiles (symbols in (a) and (b)) are symmetric, with the concentration gradient centered on the cell center. The concentration gradients predicted using RS transport coefficients (Fig. 4(a)) also show a symmetric profile that is centered close to the cell center, and hence matches the experimental data. On the contrary, the concentration gradients predicted using PNB transport coefficients (Fig. 4(b)) exhibit asymmetric profiles that are not centered on the cell center. Qualitatively, this asymmetry results from sharply varying transference numbers in a narrow concentration regime near $r=0.1$ (see Table S3, ESI + ). We note that since the TFSI $^{-}$concentration changes by $>30 \%$ in our experiment, we are able to effectively probe the transference numbers over a wide range of salt-to-polymer concentrations, which is an advantage compared to single concentrations measurements. An overlay plot of the concentration gradient highlighting the observed behavior in Fig. 4 between the experiment and the two predictions at $t=707 \mathrm{~min}$ is shown in Fig. S10 in the ESI. $\dagger$

Next, we first discuss the origin of the XPCS-measured velocity and then discuss our observations with respect to the CM predictions. Specifically, this velocity corresponds to the relative velocity of the part of the sample that gives rise to the scattering in the measured $q$-range with respect to the static cell windows; this is essentially equivalent to referencing the velocity with respect to the lithium electrodes, as these are rigidly clamped to the windows and static (see Fig. S11, ESI $\dagger$ ).

The measured $q$-range was between $q_{\min }=0.0029 \AA^{-1}$ and $q_{\max }=0.0192 \AA^{-1}$ (see the ESI $\dagger$ ), i.e. the scattering in the measured range originates from density fluctuations on length scales of $2 \pi / q \approx 300-2000 \AA$. Intuitively, this suggests that the observed scattering has its origin in heterogeneities in the network structure of the polymer-LiTFSI melt, rather than in the individual $\mathrm{TFSI}^{-}$anions, which have a size of less than $10 \AA$. This is consistent with the measured scattering intensity as a function of time. Fig. S3 (ESI $\dagger$ ) shows the transmission corrected mean scattering intensity as a function of time for the five different locations, normalized to the initial mean intensity, and Fig. S4 (ESI $\dagger$ ) shows $I(q)$ for several locations over time. We observe a trend in which the scattering intensity increases for decreasing ion concentration, whereas the scattering intensity decreases for increasing ion concentration. In the center of the channel, where the ion concentration essentially remains unchanged, the scattering intensity also does not vary significantly. This demonstrates that XPCS originates from the dynamics of the density fluctuations in the arrangement of polymer chains and that the measured velocity corresponds to the motion of the overall polymer-salt matrix with respect to the electrodes.

Interestingly, the structural properties of the LiTFSI/PEO network evolve faster during ionic mass transport compared to equilibrium conditions at open circuit before polarization. This is evident from Fig. S12 (ESI $\dagger$ ), which shows auto-correlation functions before and after cell polarization. The decay time $\left(\tau_{0}\right.$ in eqn (2)) before polarization is about one order of magnitude slower and exhibits no $\chi$-dependence, as expected. Since the decay time encodes the system's self-dynamics and is in principle independent of any velocity field, ${ }^{28}$ these observations suggest that the moving ions disrupt the polymer network, resulting in rearrangements of the network on time-scales faster than the self-dynamics at open circuit equilibrium. While the dynamics slow down during polarization, it is unclear if the altered dynamics are a result of motion of the cation or of the anion or both as a steady state was not reached (during which only cation contributions would be observed). 
On a macroscopic level, a combination of volume conservation, local and global incompressibility of the polymer, and electroneutrality imposes that the anion velocity is related to the solvent velocity via $^{19}$

$$
v_{\mathrm{TFSI}^{-}}=-\varepsilon v_{\mathrm{EO}}
$$

where $\varepsilon=\frac{V_{\mathrm{EO}} c_{\mathrm{EO}}}{V_{\mathrm{LiTFSI}} c_{\mathrm{LiTFSI}}}$, and $c_{\mathrm{EO}}$ and $c_{\mathrm{TFSI}}$ are the concentration of the solvent and salt, respectively, and $V_{\mathrm{EO}}$ and $V_{\mathrm{TFSI}}$ are the molar volumes of the solvent and salt, respectively. In other words, the polymer needs to "make space" for TFSI $^{-}$ions that would like to pile up near the positive terminal. A microscopic illustration of this phenomenon is shown in the ESI $\dagger$ (Fig. S13).

Since CM predicts the $\mathrm{TFSI}^{-}$velocity and XPCS measures the ensemble average velocity of EO monomers, to relate these, we now need to estimate the molar volumes of the EO monomers and LiTFSI salt. Assuming that the molar volumes are independent of concentration, the individual solvent and salt molar volumes can be determined by fitting the concentration dependence of the mass density ${ }^{47}$ for the molar ratio $r$ to

$$
\rho=\frac{M_{\mathrm{LiTFS}}+\left(M_{\mathrm{EO}} / r\right)}{V_{\mathrm{LiTFSI}}+\left(V_{\mathrm{EO}} / r\right)},
$$

where $M_{\mathrm{EO}}$ and $M_{\mathrm{LiTfSI}}$ are the molar masses of the EO monomer and LiTFSI of 44.05 and $287.08 \mathrm{~g} \mathrm{~mol}^{-1}$, respectively. The density data by Pesko et $a l^{25}$ is shown together with the fit in Fig. S14 (ESI $\dagger$ ), yielding molar volume derived molecular volumes of $V_{\mathrm{EO}}^{\text {molecule }}=66.4 \pm 0.73 \AA^{3}$ and $V_{\mathrm{TFSI}}^{\text {molecule }}=224 \pm 7 \AA^{3}$, values in good agreement with the numbers obtained for EO from the PEO density of $65 \AA^{3}$, and for the volume of $\mathrm{TFSI}^{-}$of $248 \AA^{3} .^{48}$ Accordingly, for $r=0.1$ we find $\varepsilon=-(2.95 \pm-0.01)$. This means that the XPCS measured velocity (of the EO ensemble average) must be multiplied by 2.95 to yield a velocity for TFSI $^{-}$. We point out that assuming a constant ratio is an approximation since the ion concentration changes as a function of $x$, and thus $r$, which would affect the conversion factor, and since there is the potential existence of ion clustering, ${ }^{49,50}$ which is not taken into account in this conversion. Since the CM predicted $\mathrm{TFSI}^{-}$ velocity is referenced with respect to the solvent velocity, ${ }^{9}$ it overestimates the velocity with respect to the fixed electrodes. This is accounted for by the conversion factor $\kappa=\frac{1}{\varepsilon}+1$ for the TFSI $^{-}$velocity derived from XPCS for a direct comparison with the anion velocities from CM. This highlights the importance of accounting for the solvent velocity when comparing predictions from concentrated solution theory to precision measurements.

Comparisons between the XPCS determined $\mathrm{TFSI}^{-}$velocities upon $0.3 \mathrm{~V}$ cell polarization and those predicted from CM using RS transport coefficients and PNB transport coefficients are shown in Fig. 5. Specifically, the velocities are shown for five different locations across the channel. While the overall predicted curves exhibit similar profiles and resemble the shape of the electrochemically measured current density, substantial differences can be observed in the evolution of the velocities over time at the different positions. Specifically, the experimental and both simulated velocity profiles show three regimes. We, somewhat arbitrarily, break them down into "slow" (e.g. experimental velocity $<50 \AA \mathrm{s}^{-1}$ at $t=300 \mathrm{~min}$ ), "medium” (e.g. experimental velocity $<100 \AA^{-1} \mathrm{~s}^{-1}$ at $t=300 \mathrm{~min}$ ), and "fast" (e.g. experimental velocity $>100 \AA \mathrm{s}^{-1}$ at $\left.t=300 \mathrm{~min}\right) \mathrm{TFSI}^{-}$velocity regimes; however, these are differently distributed across the cell depending on the transport model: for RS transport coefficients, the yellow and green location belong to the fast regime, the blue and black location to the medium regime, and the magenta to the slow regime. On the contrary, for PNB transport coefficients, the green, yellow, and blue location belong to the fast regime, the black location to the medium regime, and the magenta to the slow regime. The experimental spatial velocity variation along the cell length $(x)$ resembles the one simulated using RS transport coefficients, whereas substantial differences are observed for PNB transport coefficients. This is manifested by the fact that the variation in the difference between simulation and experiment shows significantly less spread across the different positions for the CM calculations using the RS transport coefficients as compared to the PNB transport coefficients (see Fig. S16, ESI $\dagger$ ). This is illustrated in the inset of Fig. 5(a), which shows the polarization time-averaged ratio of the XPCS determined $\mathrm{TFSI}^{-}$velocity and those obtained using CM. While there is an absolute difference between the measured and predicted velocities in both cases and the magenta location appears to be an outlier (see also Fig. S15 and S16, ESI $\dagger$ ), the position dependence shows a better match to the profiles calculated using RS transport coefficients, as evident from the fact that the black, yellow, green, and blue location exhibit ratios falling within the red bar in the inset of Fig. 5(a), whereas only the yellow and green location fall within this range for the PNB transport coefficients (see also Fig. S17, ESI $\dagger$ ). This is quantified by a standard deviation of the ratio at the black, yellow, green, and blue location of 0.08 for RS transport coefficients and 0.12 for PNB transport coefficients. Together with the results of the XAM and MD simulations below (Fig. 4), our velocity measurement results support the RS transport coefficients.

To provide a mechanistic microscopic rationalization of both the microscopic velocity measurements and macroscopic concentration polarization discussed above, we employed MD simulations (simulation box snapshot in Fig. S7a, ESI $\dagger$ ) at temperatures of $90-150{ }^{\circ} \mathrm{C}$, which predict structural and transport properties in good agreement with the experiments (Fig. S7b and S8, ESI $\dagger$ ). Specifically, we analyze $t_{\mathrm{app}}{ }^{+}$, the apparent transference number, which does not explicitly include ion-ion correlations and is extracted from self-diffusion coefficients and corresponds to pfgNMR results, as well as $t^{+}$, which includes ionic correlations with the flux under anion blocking conditions. The latter is extracted via application of Onsager reciprocal relations combined with linear response theory within the Wohde-Roling-formalism ${ }^{33}$ and is comparable to e-NMR results (see eqn (S22)-(S26) in the ESI $\dagger$ ). For $r=0.1$, our MD simulations predict $t_{\text {app }}{ }^{+}=0.17-0.20$, whereas a smaller value is found for $t^{+}=0.12-0.13$. Both of these predictions are within experimental error bars reported from pfg-NMR and e-NMR (RS transport coefficients) by Rosenwinkel et $a .^{21}$ At a higher salt concentration of $r=0.16, \mathrm{MD}$ simulations predict $t^{+}=0.17-0.20$, which suggests fairly concentration-independent transference 

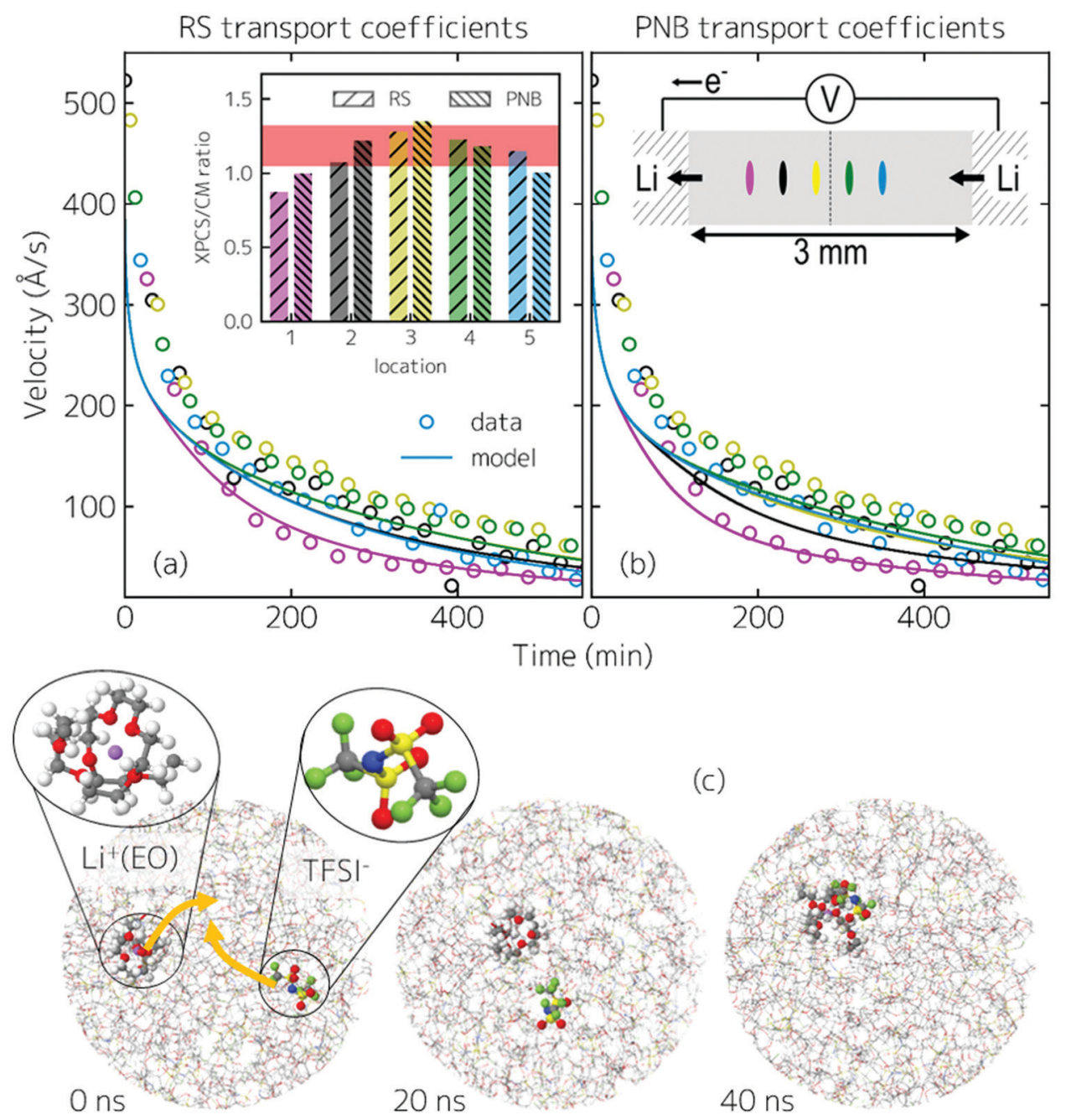

Fig. 5 Electrolyte velocities. (a) XPCS-derived TFSI ${ }^{-}$velocity (markers) for different locations within the cell (color-code corresponding to the inset in (b), i.e. $x=-0.85 \pm 0.04,-0.50 \pm 0.04,-0.15 \pm 0.04,0.20 \pm 0.04$, and $0.55 \pm 0.04 \mathrm{~mm}$, where $x=0$ corresponds to the half-way point between the electrodes indicated by the dashed line) measured upon constant voltage ( $0.3 \mathrm{~V})$ polarization of the Li/LiTFSI-PEO/Li symmetric cell of a channel length of $3 \mathrm{~mm}$ (see the inset in (b)) as a function of time, and the TFSI ${ }^{-}$velocity predicted from the continuum model under conditions identical to the experiment using RS transport coefficients ${ }^{21}$ (lines). The inset shows the polarization time-averaged $(200<t<400$ min) ratio between the XPCS measured and CM model simulation TFSI ${ }^{-}$velocities for the five different locations. The red bar is centered around the mean ratio (1.19) between the experiment and both simulations at the black, yellow, green, and blue locations and has a width of \pm 1.5 times the standard deviation of 0.10 . (b) Same as (a) using PNB transport coefficients. ${ }^{25,34}$ The colored regions in the inset correspond to the measured locations in the channel and are color coded with the velocity measurements (markers) and CM predictions (lines). We note that velocities after polarization times greater than about 550 minutes were not resolvable. (c) MD trajectories showing anticorrelated motion (movement towards each other as indicated by the orange arrows) of a representative $\mathrm{Li}^{+}(\mathrm{EO})_{6}$ complex and TFSI${ }^{-}$(white: $\mathrm{H}$, grey: C, red: O, magenta: Li, green: $\mathrm{F}$, blue: $\mathrm{N}$, yellow: S).

numbers around 0.2, consistent with the RS transport coefficients, supporting our experimental findings. To rationalize the microscopic origin of the predicted transference numbers, we consider the degree of correlation of the $\mathrm{Li}^{+}$and $\mathrm{TFSI}^{-}$displacements at $r=$ 0.1. As detailed in the ESI, $\uparrow$ we find moderate anti-correlated motion and the following picture emerges: at short time scales (i.e. lower than the residence time of $\mathrm{Li}^{+}$with a polymer segment), the $\mathrm{Li}^{+}(\mathrm{EO})_{6}$ complex partially diffuses in the direction opposite to the $\mathrm{TFSI}^{-}$ anion, somewhat resembling the anticorrelation observed in the tetraglyme-LiTFSI molecular system. ${ }^{13}$ This anticorrelation of the $\mathrm{Li}^{+}(\mathrm{EO})_{6}$ solvate and TFSI ${ }^{-}$, which is illustrated in Fig. 5(c), explains on a microscopic level why $t^{+}$is lower than $t_{\text {app }}{ }^{+}$. This observation closes the gap between microscopic and macroscopic transport phenomena by suggesting that the lower transference numbers are a result of anticorrelated motion on the nanoscale, which in turn result in significant mass transport of $\mathrm{TFSI}^{-}$and the electrolyte, which then are also anti-correlated.

\section{Conclusions}

We illustrated a novel methodology combining several measurement techniques and simulations to provide quantitative length-scale bridging insight into ion transport in electrolytes. Specifically, we combined synchrotron X-rays to retrieve 
with high accuracy local electrolyte velocities via XPCS and global concentration gradients via XAM with continuum modelling supported by molecular scale insight provided by MD simulations. To the best of our knowledge, our velocity measurements represent the first spatially resolved direct measurements of ion velocities as a function of time in an electrochemical system. The strength of this approach lies in the opportunity to directly compare physically measured mass transport effects upon cell polarization with those calculated from concentrated solution theory using transport coefficients as input values. Together with MD simulations, this method not only allows us to quantify transport parameters such as the transference number, but also provides atomistic scale insight into the origin of the obtained values. We applied our approach to an exemplar LIB polymeric electrolyte, LiTFSI-PEO, and shed light onto the concentration dependence of the transference numbers in this system, which is debated by researchers; the outlined procedure can also be applied to aqueous and nonaqueous liquid electrolytes when convection can be avoided, as well as to more complex electrolytes, such as composite electrolytes or those infiltrated with fillers for the sake of improved ionic conductivity. ${ }^{51,52}$ Such direct insight paves the way for understanding of ion transport in general, and the proposed methodology can provide novel insight not only into ion transport in polymeric electrolytes but also in liquid electrolytes and electrode materials, as well as separation membranes or polymer electrolyte membranes for fuel cells. Finally, we argue that the many orders increase in coherent flux at diffraction limited storage rings will enable probing transport phenomena at the microscopic level by XPCS to be applied to other systems, taking advantage of the intensity and penetration power of next generation X-ray beams.

\section{Author contributions}

Conceptualization: HGS, CJT, HHK, DMM, BH, OB, SKS, VS, MFT. Methodology: HGS, CJT, HHK, DMM, BH, OB, SKS, VS, MFT. Software: HGS, CJT, HHK, DMM, BH, OB. Validation: HGS, CJT, HHK, DMM, BH, CC, SN, EMD, YC, BR, FZ, JW, OB, SKS, VS, MFT. Formal analysis: HGS, CJT, HHK, DMM, BH, OB. Investigation: HGS, CJT, HHK, DMM, BH, CC, SN, EMD, YC, BR, FZ, JW, OB, SKS, VS, MFT. Resources: HGS, CJT, HHK, DMM, BH, CC, SN, EMD, YC, BR, FZ, JW, OB. Data curation: HGS, CJT, HHK, DMM, BH, OB. Writing - original draft preparation: HGS, CJT, HHK, DMM, OB. Writing - review and editing: HGS, CJT, HHK, DMM, BH, CC, SN, EMD, YC, BR, FZ, JW, OB, SKS, VS, MFT. Visualization: HGS, OB. Supervision: HGS, CJT, OB, SKS, VS, MFT. Project administration: HGS, CJT, DMM. Funding acquisition: SKS, OB, VS, MFT.

\section{Funding}

This work was supported as part of the Joint Center for Energy Storage Research, an Energy Innovation Hub funded by the U.S. Department of Energy, Office of Science, Basic Energy Sciences.
This research used resources of the Advanced Photon Source, a U.S. Department of Energy (DOE) Office of Science User Facility operated for the DOE Office of Science by Argonne National Laboratory under Contract No. DE-AC02-06CH11357. The research of SKS and $\mathrm{BH}$ was supported by Grant DESC0003678 from Division of Basic Energy Sciences, U.S. Department of Energy.

\section{Conflicts of interest}

The authors declare no competing interests.

\section{Acknowledgements}

We are extremely grateful to Nitash Balsara for many fruitful discussions and his insightful constructive comments. It is a pleasure to thank Mark Sutton and Alec Sandy for discussions. The assistance of Ross Arthur and Geovanni Gordon in the design and construction of the electrochemical cell is gratefully acknowledged, as is the help of Karim Lhoste during experiments at ID10. The authors acknowledge ESRF for providing beamtime for the proposal MA3290 on ID10.

\section{References}

1 E. Gouaux and R. Mackinnon, Science, 2005, 310, 1461-1465.

2 K. Xiao, L. Chen, R. Chen, T. Heil, S. D. C. Lemus, F. Fan, L. Wen, L. Jiang and M. Antonietti, Nat. Commun., 2019, 10, $1-7$.

3 K. Xiao, L. Jiang and M. Antonietti, Joule, 2019, 3, 2364-2380.

4 D. Cohen-Tanugi and J. C. Grossman, Nano Lett., 2012, 12, 3602-3608.

5 D. T. Hallinan and N. P. Balsara, Annu. Rev. Mater. Res., 2013, 43, 503-525.

6 H. Sun, J. Zhu, D. Baumann, L. Peng, Y. Xu, I. Shakir, Y. Huang and X. Duan, Nat. Rev. Mater., 2019, 4, 45-60.

7 S. Shi, J. Gao, Y. Liu, Y. Zhao, Q. Wu, W. Ju, C. Ouyang and R. Xiao, Chin. Phys. B, 2016, 25, 018212.

8 N. P. Balsara and J. Newman, J. Electrochem. Soc., 2015, 162, A2720-A2722.

9 J. Newman and K. E. Thomas-Alyea, Electrochemical systems, John Wiley \& Sons, 2012.

10 I. Villaluenga, D. M. Pesko, K. Timachova, Z. Feng, J. Newman, V. Srinivasan and N. P. Balsara, J. Electrochem. Soc., 2018, 165, A2766-A2773.

11 D. J. Brooks, B. V. Merinov, W. A. Goddard, B. Kozinsky and J. Mailoa, Macromolecules, 2018, 51, 8987-8995.

12 O. Borodin and G. D. Smith, Macromolecules, 2006, 39, 1620-1629.

13 D. Dong, F. Sälzer, B. Roling and D. Bedrov, Phys. Chem. Chem. Phys., 2018, 20, 29174-29183.

14 K. D. Fong, J. Self, K. M. Diederichsen, B. M. Wood, B. D. Mccloskey and K. A. Persson, ACS Cent. Sci., 2019, 5, 1250-1260.

15 D. T. Hallinan, I. Villaluenga and N. P. Balsara, MRS Bull., 2018, 43, 759-767. 
16 S. B. Aziz, T. J. Woo, M. F. Z. Kadir and H. M. Ahmed, J. Sci., 2018, 3, 1-17.

17 K. M. Diederichsen, E. J. McShane and B. D. McCloskey, ACS Energy Lett., 2017, 2, 2563-2575.

18 M. Doyle, T. F. Fuller and J. Newman, Electrochim. Acta, 1994, 39, 2073-2081.

19 A. Nyman, M. Behm and G. Lindbergh, Electrochim. Acta, 2008, 53, 6356-6365.

20 D. B. Shah, H. K. Kim, H. Q. Nguyen, V. Srinivasan and N. P. Balsara, J. Phys. Chem. C, 2019, 123, 23872-23881.

21 M. P. Rosenwinkel and M. Schönhoff, J. Electrochem. Soc., 2019, 166, A1977-A1983.

22 J. Evans, C. A. Vincent and P. G. Bruce, Polymer, 1987, 28, 2324-2328.

23 P. G. Bruce and C. A. Vincent, J. Electroanal. Chem., 1987, 225, 1-17.

24 D. M. Pesko, S. Sawhney, J. Newman and N. P. Balsara, J. Electrochem. Soc., 2018, 165, A3014-A3021.

25 D. M. Pesko, K. Timachova, R. Bhattacharya, M. C. Smith, I. Villaluenga, J. Newman and N. P. Balsara, J. Electrochem. Soc., 2017, 164, E3569-E3575.

26 G. Grübel, A. Madsen and A. Robert, Soft Matter Characterization, 2008, pp. 953-995, DOI: 10.1007/978-1-40204465-6_13.

27 M. Sutton, C. R. Phys., 2008, 9, 657-667.

28 F. Livet, F. Bley, F. Ehrburger-Dolle, I. Morfin, E. Geissler and M. Sutton, J. Synchrotron Radiat., 2006, 13, 453-458.

29 B. J. Berne and R. Pecora, Dynamic light scattering: with applications to chemistry, biology, and physics, Courier Corporation, 2000.

30 F. Ehrburger-Dolle, I. Morfin, F. Bley, F. Livet, G. Heinrich, S. Richter, L. Piché and M. Sutton, Macromolecules, 2012, 45, 8691-8701.

31 J. G. Ulbrandt, M. G. Rainville, C. Wagenbach, S. Narayanan, A. R. Sandy, H. Zhou, K. F. Ludwig and R. L. Headrick, Nat. Phys., 2016, 12, 794-799.

32 J. R. M. Lhermitte, M. C. Rogers, S. Manet and M. Sutton, Rev. Sci. Instrum., 2017, 88, 015112.

33 F. Wohde, M. Balabajew and B. Roling, J. Electrochem. Soc., 2016, 163, A714-A721.
34 D. M. Pesko, Z. Feng, S. Sawhney, J. Newman, V. Srinivasan and N. P. Balsara, J. Electrochem. Soc., 2018, 165, A3186-A3194.

35 W. Gorecki, M. Jeannin, E. Belorizky, C. Roux and M. Armand, J. Phys.: Condens. Matter, 1995, 7, 6823-6832.

36 K. Pożyczka, M. Marzantowicz, J. R. Dygas and F. Krok, Electrochim. Acta, 2017, 227, 127-135.

37 C. Xu, B. Sun, T. Gustafsson, K. Edström, D. Brandell and M. Hahlin, J. Mater. Chem. A, 2014, 2, 7256-7264.

38 C. F. N. Marchiori, R. P. Carvalho, M. Ebadi, D. Brandell and C. M. Araujo, Chem. Mater., 2020, 32(17), 7237-7246.

39 J. Alvarado, M. A. Schroeder, T. P. Pollard, X. Wang, J. Z. Lee, M. Zhang, T. Wynn, M. Ding, O. Borodin, Y. S. Meng and K. Xu, Energy Environ. Sci., 2019, 12, 780-794.

$40 \mathrm{H}$. Yildirim, J. B. Haskins, C. W. Bauschlicher and J. W. Lawson, J. Phys. Chem. C, 2017, 121, 28214-28234.

41 I. Rey, J. L. Bruneel, J. Grondin, L. Servant and J. C. Lassegues, J. Electrochem. Soc., 1998, 145, 3034-3042.

42 C. Brissot, M. Rosso, J. N. Chazalviel and S. Lascaud, J. Power Sources, 2001, 94, 212-218.

43 C. Brissot, M. Rosso, J. N. Chazalviel and S. Lascaud, J. Electrochem. Soc., 1999, 146, 4393-4400.

44 P. Georén, J. Adebahr, P. Jacobsson and G. Lindbergh, J. Electrochem. Soc., 2002, 149(8), A1015-A1019.

45 J. Newman and T. W. Chapman, AIChE J., 1973, 19, 343-348. 46 D. Takamatsu, A. Yoneyama, Y. Asari and T. Hirano, J. Am. Chem. Soc., 2018, 140, 1608-1611.

47 P. Georen and G. Lindbergh, Electrochim. Acta, 2001, 47, 577-587.

48 R. L. Gardas and J. A. P. Coutinho, Fluid Phase Equilib., 2008, 263, 26-32.

49 N. Molinari, J. P. Mailoa and B. Kozinsky, Chem. Mater., 2018, 30, 6298-6306.

50 H.-K. Kim, N. P. Balsara and V. Srinivasan, J. Electrochem. Soc., 2020, 167, 110559.

51 J.-F. Wu and X. Guo, J. Mater. Chem. A, 2019, 7, 2653-2659.

52 W. Tang, S. Tang, X. Guan, X. Zhang, Q. Xiang and J. Luo, Adv. Funct. Mater., 2019, 29, 1900648.

53 A. R. Sandy, L. B. Lurio, S. G. J. Mochrie, A. Malik, G. B. Stephenson, J. F. Pelletier and M. Sutton, J. Synchrotron Radiat., 1999, 6, 1174-1184. 